\title{
Parametric Evaluation of Wobble-Yoke Stirling Engine State Space Model
}

\author{
Ermira Junita Abdullah ${ }^{1, *}$ and Rick Kit Lee $^{1}$ \\ ${ }^{1}$ Department of Aerospace Engineering, Faculty of Engineering, Universiti Putra Malaysia, 43400, \\ Serdang, Selangor, Malaysia
}

\begin{abstract}
A conceptual design of Stirling engine suitable for onboard aircraft application is the motivation behind this study. The aim is to evaluate the suitability of the system to be integrated in aircraft as part of energy contributor by recycling the waste thermal energy. A parametric evaluation of an established Wobble-Yoke Stirling Engine state space model was performed to determine a suitable pre-compensator design that is able to produce the highest power output given the size and mass constraint. Simulation was performed using MATLAB in order to analyze the dynamic properties of the engine as a closed-loop system, with temperature, pressure and damping coefficient acting as controlling parameters. The parametric evaluation was conducted to determine the effect of assemble mass of piston, maximum displacement of piston and viscous damping coefficient to the performance of the system. The methodology can be used in the conceptual design process in order to determine the sizing and power output of the stirling engine system.
\end{abstract}

\section{Introduction}

Stirling engine has a good potential to be used as a thermal recovery mechanism. It is a heat engine that is operated by a cyclic compression and expansion of air or other gas (the working fluid) at different temperatures, such that there is a net conversion of heat energy to mechanical work [1]. The idea of this study is to incorporate the Stirling engine into aircraft to recycle the wasted heat energy produced by the engine under the cabin, within the wind chamber or aft pylon into useful mechanical energy that can oscillate the assembled piston within to generate ultimate electrical energy for the avionic systems. A study has shown how much waste heat generated by the engine nearby the aft pylon that caused its environment temperature to rise by $200^{\circ} \mathrm{C}$. The maximum temperature of $580^{\circ} \mathrm{C}$ can be detected at its outer surface and minimum of $350^{\circ} \mathrm{C}$ can be detected at inner surface [2]. Yet, the temperature farther than the engine is cooler and the difference in temperature can be fully utilized by using Stirling engine to produce sufficient energy for avionics systems or simple mechanical action in aircraft.

\footnotetext{
* Corresponding author: ermira@upm.edu.my
} 
Stirling engine can be used to recycle a lot of energy waste especially thermal waste that is a side or waste product during the chemical reactions which take place anywhere, anytime around us. Furthermore, Stirling engine can produce energy in condition of low level of noise and vibration when converting heat energy to shaft power [3]. However, it is still not widely used due to critical issues including unstable closed-loop system. The performance and data of Stirling engine is typically not accessible to the public and only a few Stirling engines being patented until today.

For the application on board aircraft, there are two main issues which are uncontrollable shifting of power-output and insufficient power output. The uncontrollable shifting of poweroutput is mainly due to uncontrollable pressure elevation caused by high fluctuation of differential temperature or intentional acceleration or deceleration [4]. The unstable thermodynamic parameters caused highly unstable movement of piston in the Stirling engine, thus the power output will fluctuate accordingly. A beta type engine prototype that weighed 360 grams was built and attached to modified Super Malibu radio control glider can only afford a gross take-off weight of $1 \mathrm{~kg}$ [5]. It produced only 20 watts of power which would not enough to generate useful mechanical nor electrical output.

The study presented here seeks to investigate dynamic modelling and control of Wobble-Yoke Stirling engine by simulating linear dynamics of the engine in-order to evaluate its suitability and capability to be renewable energy source on-board aircraft. The present work will focus on problem of its unstable closed-loop piston and control its power output more efficiently using its dynamic model. Parametric studies were conducted using mathematical modelling in MATLAB with state-space block in Simulink in order to approximate the power output for different design constraints. The method is useful in the conceptual design process for sizing purposes.

\section{Dynamic Model}

A general model that relies and depends upon the change in time is known as the dynamic model. It allows complex interactions between few variables or parameters over time. Dynamic modelling of a subsystem or physical system could describe the behaviour of the system. Many dynamic models have been developed for the Stirling engine and one such model for the Wobble Yoke Stirling engine [6] is used here to evaluate it's dynamics performance. The second-order differential equation has been derived and presented in the reference [6]. The engine can be considered as an unstable linear closed-loop systems due to the mass-spring behaviour of the working gases where the state feedback is given by the pressure variations in the pistons. By using linear control technique a pre-compensator can be incorporated to ensure the stability of the pistons displacements.

The dynamic model can be represented as a state space model where the state of a system is described by the state variables, $x$, and the input vector, $u$. Thus, the system can be written in the following form:

$$
\dot{\boldsymbol{x}}=\mathbf{A} \boldsymbol{x}+\mathbf{B} \boldsymbol{u}
$$

where the matrix $\mathrm{A}$ is an $\mathrm{n} \times \mathrm{n}$ square matrix and $\mathrm{B}$ is an $\mathrm{n} \times \mathrm{m}$ matrix. Relationship between the outputs of a linear system to the state variables and the input signals is given by

$$
y=\mathrm{C} x
$$

where $y$ is the set of output signals expressed in column vector. For the Wobble Yoke Stirling engine, the matrices are defined as follows: 


$$
\begin{gathered}
\mathbf{A}=\left[\begin{array}{cccc}
0 & 0 & 1 & 0 \\
0 & 0 & 0 & 1 \\
-\frac{k_{p_{1}}}{m} & 0 & -\frac{b_{p_{1}}}{m} & 0 \\
0 & -\frac{k_{p_{2}}}{m} & 0 & -\frac{b_{p_{2}}}{m}
\end{array}\right] \\
\mathbf{B}=\left[\begin{array}{ccc}
0 & 0 & 0 \\
0 & 0 & 0 \\
\frac{\left(A_{p}-A_{r}\right)}{m} & 0 & -\frac{A_{p}}{m} \\
-\frac{A_{p}}{m} & \frac{\left(A_{p}-A_{r}\right)}{m} & 0
\end{array}\right] \\
C=\left[\begin{array}{cccc}
1 & 0 & 0 & 0 \\
0 & 1 & 0 & 0 \\
0 & 0 & 1 & 0 \\
0 & 0 & 0 & 1
\end{array}\right] \\
\boldsymbol{u}=-\mathbf{K} \boldsymbol{x}
\end{gathered}
$$

where $A_{p}$ is the piston area, $A_{r}$ is the piston rod area, $k_{p i}$ is the piston spring constant and $b_{p i}$ is the damping coefficient. The system is self-excited through the input term $\boldsymbol{u}$, which depends on initial pressure and temperature conditions to induce the subsequent operation of engine. The block diagram in Figure 1 shows the state-space block with input, which could be further manipulated by altering the parameters of the system.

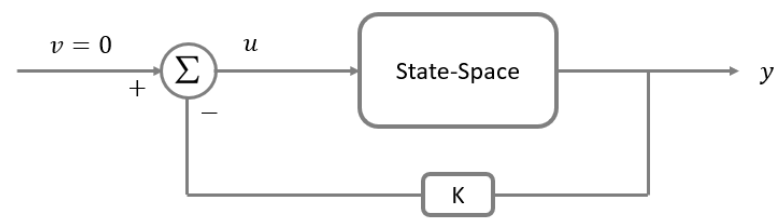

Fig. 1. Block Diagram of a Closed-Loop System.

The damping coefficient used here is $b_{p_{i}}=10 \frac{\mathrm{Ns}}{\mathrm{m}}$ as referred to prior work using this model $[7,8]$. At this value of damping coefficient, the system has two positive real parts of the pole which means that the system is unstable. Theoretically a stable cyclic steady-state of the Wobble-Yoke Stirling engine occurs when $b_{p_{i}}=143 \frac{\mathrm{Ns}}{\mathrm{m}}$. Existence of precompensator $\boldsymbol{v}(\boldsymbol{x})$ was possible to alter the system's input, $\boldsymbol{u}$ [9]. State feedback to new input:

$$
u(x)=-K x+v(x)
$$

New control input with all vector state $\boldsymbol{x}$ were measured:

$$
u=u_{r}-\overline{\mathrm{K}}\left(x-x_{r}\right)
$$

A proper choice of pole placement can alter the value of $b_{p_{i}}$ and thus affect the value of $\overline{\mathrm{K}}$ in the end as shown in the pre-compensator system block diagram in Figure 2. 


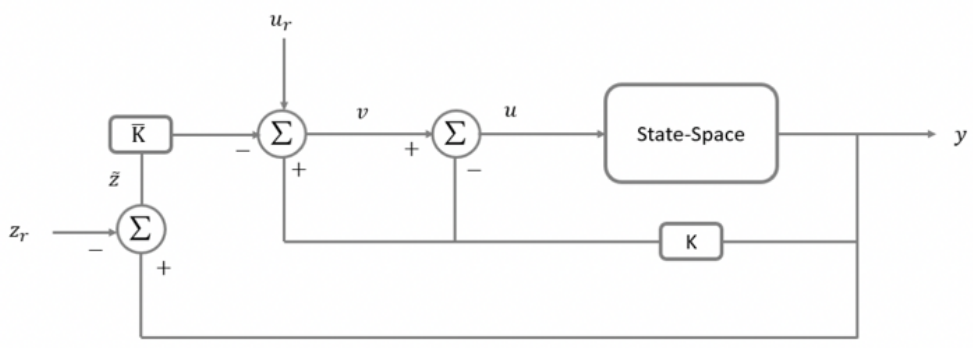

Fig. 2. Pre-compensator block diagram

For desired pole design, an appropriate gain, $\overline{\mathrm{K}}$ has to be developed. Firstly, the damping coefficient, $b_{p_{d}}$, that could provide poles and zeroes on the left-hand side (LHS) of pole-zero diagram can be selected as it acts as a controller. Then, the eigenvalues of the new matrix A can be found as the damping coefficient is related to the state matrix A.

$$
\mathbf{A}_{\text {new }}=\left[\begin{array}{cccc}
0 & 0 & 1 & 0 \\
0 & 0 & 0 & 1 \\
-\frac{k_{p_{1}}}{m} & 0 & -\frac{b_{p_{d}}}{m} & 0 \\
0 & -\frac{k_{p_{2}}}{m} & 0 & -\frac{b_{p_{d}}}{m}
\end{array}\right]
$$

Then, the eigenvalues of $\mathbf{A}_{\text {new }}$ are placed into the desired pole

$$
\text { pole, } \mathrm{p}=\left[\operatorname{eig}\left(A_{\text {new }}\right)_{1}, \text { eig }\left(A_{\text {new }}\right)_{2}, \text { eig }\left(A_{\text {new }}\right)_{3}, \text { eig }\left(A_{\text {new }}\right)_{4}\right]
$$

Thus, the matrix of $A_{\text {new }}, B$ and $\mathrm{p}$ are placed into the appropriate gain matrix, $\overline{\mathrm{K}}$.

$$
\overline{\mathrm{K}}=\operatorname{place}\left(A_{\text {new }}, B, p\right)
$$

\section{Methodology}

Figure 3 shows the algorithm for the modelling used in this study. The first step was to select the parameters value of the systems which include its physical parameters, thermodynamic parameters and parameters used for the parametric evaluation. Then, the equations and constants for state feedback are defined which eventually will indicate the system is selfexcited and it is a closed-looped system. After defining the state feedback, the parameters used for the parametric evaluation are defined, which was the damping coefficient, $b_{p i}$, assembled mass of piston rods, $m$ and maximum displacement of piston, $z_{\max }$. These three parameters would then be evaluated with established dynamic state-space equation through pole-zero plot using MATLAB. If the plot shows that the poles are on the LHS, the system is considered stable and if it is on the RHS, the system is considered unstable and the system proceeds to repeat the step of inserting suitable value of the selected parameters.

Next, the value of the desired damping coefficient is input to the system. This eventually becomes the key parameter for the pre-compensator design. Once the eigenvalues are produced, the desired pole values can be used to produce a new state matrix and input matrix for the state-space equation. Eventually, an appropriate gain matrix is developed to build a complete dynamic system of Stirling engine. Finally, the desired parameters such as torque and power are extracted for further analysis. 


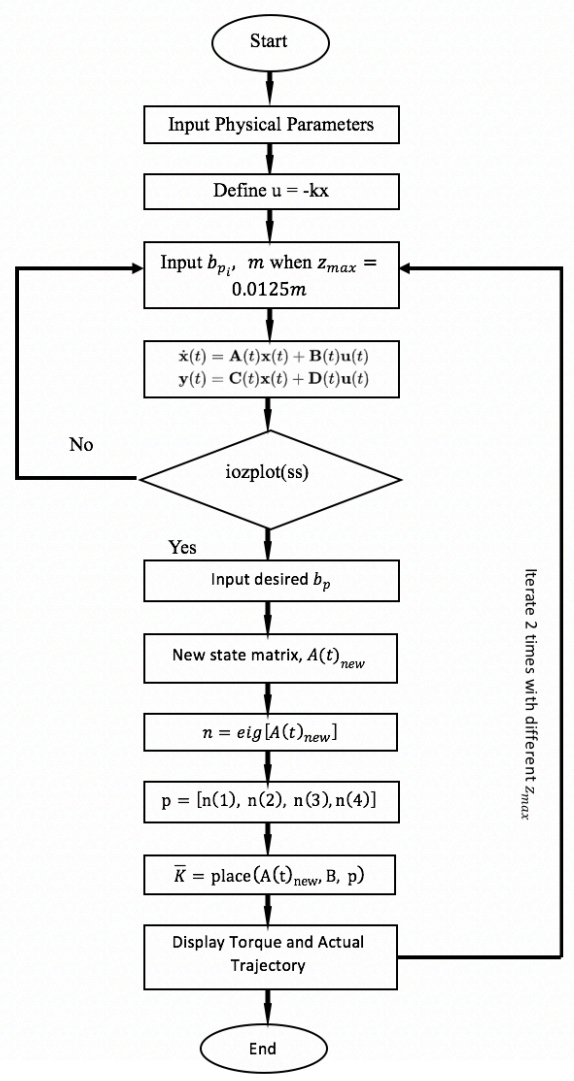

Fig. 3. Algorithm for the parametric evaluation

The parametric study was conducted to evaluate the parameters, and to identify the design constraints. The free body diagram of the system being evaluated is as shown in Figure 4. The goal was to determine the effects of the parameters on the engine's performances with the values listed in Table 1.

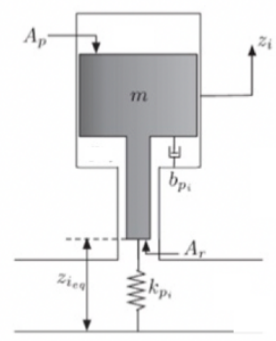

Case 1: FBD for $0.0125 \mathrm{~m}$ Piston

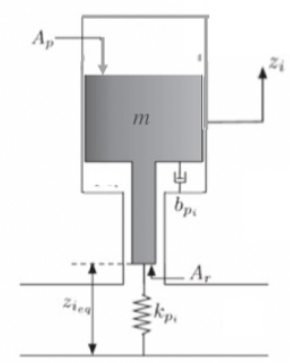

Case 2: FBD for $0.0250 \mathrm{~m}$ Piston

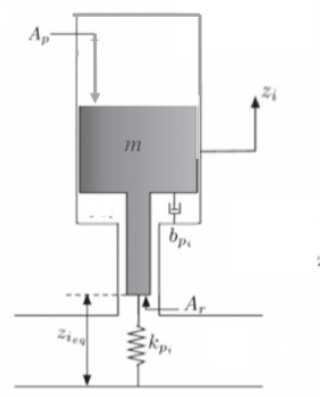

Case 3: FBD for $0.0375 \mathrm{~m}$ Piston

Fig. 4. Free body diagram of the system with different maximum piston displacement 
Table 1. Parameters variation

\begin{tabular}{|c|c|c|c|}
\hline \multirow{3}{*}{ Case } & \multicolumn{3}{|c|}{$T_{k}=300 \mathrm{~K}, \quad T_{h}=473.15 \mathrm{~K}$} \\
\hline & $m=0.025 \mathrm{~kg}$ & $m=0.1 \mathrm{~kg}$ & $m=0.4 \mathrm{~kg}$ \\
\hline & \multicolumn{3}{|c|}{ Maximum Piston Displacement, $z_{\max } / \mathrm{m}$} \\
\hline 1 & \multicolumn{3}{|c|}{0.0125} \\
\hline 2 & \multicolumn{3}{|c|}{0.0250} \\
\hline 3 & \multicolumn{3}{|c|}{0.0375} \\
\hline
\end{tabular}

The prototype used in the simulation has a default value of $b_{p_{i}}=\frac{10 N}{m^{2}}$, where the system is behaving as unstable cyclic process. By ignoring the effect of thermodynamics, the Stirling engine should work in perfect cyclic steady state, when the control characteristic has two imaginary roots and two negative real roots on the left-hand side (LHS) of the s-plane. For the model used here, the situation occurred only when $b_{p_{i}}=\frac{143 N}{m^{2}}$ [10]. Thus, the damping coefficient is the key parameter to determine the system's stability by designing desired-pole placement. Due to the instability of the system when $b_{p_{i}}=\frac{10 \mathrm{~N}}{\mathrm{~m}^{2}}$, further investigation was conducted only for $b_{p_{i}}=\frac{143 \mathrm{~N}}{m^{2}}$.

The piston assembly mass, $m$ could affect the movement of vertical displacement, which directly affects the efficiency of energy and power output. Newton first law states that object tends to stay at its initial motion, hence, a greater mass could induce a great inertia causing vertical displacement, $z_{i}$ to be affected. In addition, the maximum displacement, $z_{\max }$ could highly affect the torque generation thus manipulating power output. Hence, it became a key parameter to generate an optimum power output with stable cycle state of the system. The working mean pressure was not selected in the parametric evaluation due to its proportionality towards the change of temperature and amount of inert gases. The hot-end and cold-end temperature was designed to be $473.15 \mathrm{~K}$ and $300 \mathrm{~K}$ respectively, hence they are constant throughout the simulation.

The implementation or assembly of a set of filters and compensators into a system that controls the plant is knowns as controller. The controller in the Wobble-Yoke Stirling engine consists of a pre-compensator in the state-space system. An appropriate gain matrix, $\bar{K}$, is the main part of the pre-compensator which is affected by the desired pole-placement. Then, the desired pole can be manipulated or controlled by the eigenvalue of the damping coefficient. In short, the damping coefficient, $b_{p_{i}}$ will act as the main controller of the system.

\section{Results}

A sample simulation of the piston displacement is shown in Figure 5 where $b_{p_{i}}=\frac{143 \mathrm{Nm}}{\mathrm{s}}$, $m=0.4 \mathrm{~kg}$ and $z_{\max }=0.0125 \mathrm{~m}$. It shows that the actual trajectory follows closely the input trajectory with minimal steady state error. 


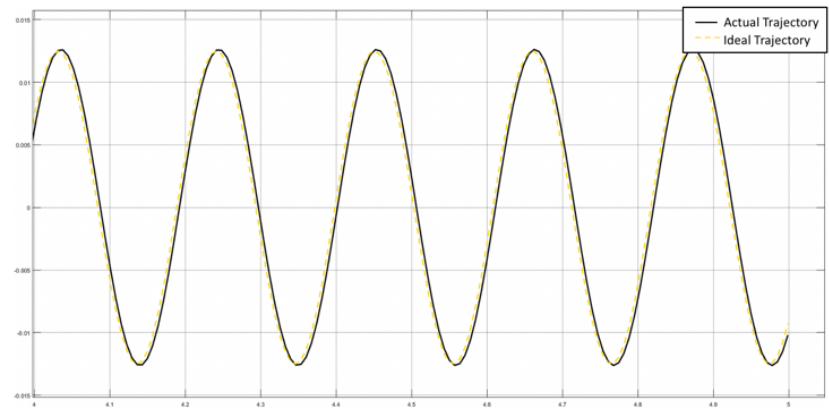

Fig. 5. Piston displacement when $b_{p_{i}}=\frac{143 \mathrm{Nm}}{\mathrm{s}}, m=0.4 \mathrm{~kg}$ and $z_{\max }=0.0125 \mathrm{~m}$.

The torque and power outputs for the simulation are summarized and presented in Table 2 - 4. For Case 1, the power output was the highest when the piston assembly mass was $0.4 \mathrm{~kg}$. Power output was $\sim 150 \%$ greater compared to power output of piston assembly mass, $0.025 \mathrm{~kg}$. This was due to the greater mass that produced a greater moment of inertia and momentum that could raise the value of torque, eventually contributing to higher power output where the power output indicates the rate of change of torque. For Case 2, the power generated was still highest when assembly mass of piston was $0.4 \mathrm{~kg}$. Power output was $\sim 200 \%$ or higher compared to power output of piston assembly mass, $0.025 \mathrm{~kg}$. For Case 3 , the same phenomena occurred as discussed in Case 1 and 2 where the highest power output produced by greatest mass. Compared to Case 1, the power output increased linearly with piston displacement and subsystem mass. This was due to higher torque produced when piston had more stretching displacement to increase crank angle and more space Thus, it could be assumed that the power output could be further amplified by enlarging the sizing of system within the constraint.

Table 2. Case 1 : Maximum Piston Displacement, $z_{\max }=0.0125 \mathrm{~m}$

\begin{tabular}{|c|c|c|}
\hline $\begin{array}{c}\text { Mass of piston } \\
\text { subsystem, } \\
\mathrm{kg}\end{array}$ & $\begin{array}{c}\text { Average } \\
\text { Torque } \\
(\mathrm{Nm})\end{array}$ & $\begin{array}{c}\text { Power Output } \\
\text { (W) }\end{array}$ \\
\hline 0.025 & 0.515 & 15.45 \\
\hline 0.1 & 2.20 & 66.0 \\
\hline 0.4 & 8.31 & 249.30 \\
\hline
\end{tabular}

Table 3. Case 2: Maximum Piston Displacement, $z_{\max }=0.025 \mathrm{~m}$

\begin{tabular}{|c|c|c|}
\hline $\begin{array}{c}\text { Mass of piston } \\
\text { subsystem, } \\
\mathrm{kg}\end{array}$ & $\begin{array}{c}\text { Average } \\
\text { Torque } \\
(\mathrm{Nm})\end{array}$ & $\begin{array}{c}\text { Power Output } \\
\text { (W) }\end{array}$ \\
\hline 0.025 & 1.074 & 32.22 \\
\hline 0.1 & 4.12 & 123.6 \\
\hline 0.4 & 16.61 & 484.8 \\
\hline
\end{tabular}


Table 4. Case 3 : Maximum Piston Displacement, $z_{\max }=0.0375 \mathrm{~m}$

\begin{tabular}{|c|c|c|}
\hline $\begin{array}{c}\text { Mass of piston } \\
\text { subsystem, } \\
\mathrm{kg}\end{array}$ & $\begin{array}{c}\text { Average } \\
\text { Torque } \\
\text { (Nm) }\end{array}$ & $\begin{array}{c}\text { Power Output } \\
\text { (W) }\end{array}$ \\
\hline 0.025 & 1.55 & 46.5 \\
\hline 0.1 & 6.18 & 185.4 \\
\hline 0.4 & 24.91 & 747.3 \\
\hline
\end{tabular}

The results show that different combination of assemble mass of piston and maximum displacement of piston will produce varying power output. With the established relationship of these parameters, optimization can be conducted given the design constraints on board aircraft such the weight and space available in order to produce the optimized power output from the engine.

\section{Conclusion}

A method to estimate the sizing and power output produced by a Wobble Yoke Stirling engine using state space modelling has been explored to be considered in the conceptual design process of renewable energy system suitable for aerospace application. The parametric evaluation was conducted to determine the effect of assemble mass of piston, maximum displacement of piston and viscous damping coefficient to the performance of the system, with the available temperature range available on board aircraft.

The simulation shows that the piston performed stable oscillation when the damping coefficient was $b_{p_{i}}=143 \frac{\mathrm{Nm}}{\mathrm{s}}$. The sizing of piston can be further enlarged to $0.035 \mathrm{~m}$ or above for higher power output. A pre-compensator that act as a controller with damping coefficient, $b_{p_{i}}$ as key parameter. Case 3 with assemble mass of piston, $0.4 \mathrm{~kg}$ produced the highest torque output of $747 \mathrm{~W}$ at $b_{p_{i}}=143 \frac{\mathrm{Nm}}{\mathrm{s}}$. In order to produce a Stirling engine with critical mass and size constraint such as for aerospace application, this parametric evaluation can be used to determine the power output. Furthermore, the damping coefficient can be tuned to achieve the desire output, as long as the system remains stable.

Future work to be considered is optimization of the performance of kinematic WobbleYoke Stirling Engine, including estimation of the actual energy production of the Stirling engine and its efficiency according to aircraft environment's suitability. The modelling of subsystem, regenerator can be included so the parameters such as pressure, temperature and more accurate dead space volume affects the performances of system.

\section{References}

1. G. Walker, Stirling Engines. Oxford: Clarendon Press (1980)

2. A. Elefsiniotis, N. Kokorakis, T. Becker, U. Schmid, A Novel High-temperature Aircraft-specific Energy Harvester Using PCMs and State of the art TEGs. Materials Today: Proceedings, 2, 2 (2015) 
3. S. P. Stolyarov, A. S. Stolayrov, Stirling Generators: Challenges and Opportunities, Russian Electrical Enginineering, 88 (2017)

4. N. Naddaf, Stirling Engine Cycle Efficiency, Bachelor Degree Thesis, University of Applied Sciences (2012)

5. R. McConaghy, A Hot Aero Engine, Model Engineer. 176 (1996)

6. E. Garcia-Canseco, J. M. A. Scherpen, M. Kuindeserma, Modeling for Control of a Kinematic Wobble-Yoke Stirling Engine, International Symposium on Nonlinear Theory and its Applications (NOLTA 2009), Sapporo, Japan (2009)

7. H. Karabalut, Dynamic Analysis of a Free Piston Stirling Engine Working with Closed and Open Thermodynamic Cycles, Renewable Energy, 36, 6 (2011)

8. J. Riofrio, K. Al-Dakkan, M. Hofacker, E. Barth, Control-based Design of Free- piston Stirling Engines, American Control Conference 2008, Seattle, Washington, USA (2008)

9. A. Alvarez-Aguirre, E. Garcia-Canseco, J. M. A. Scherpen, Linear Dynamics and Control of a Kinematic Wobble-Yoke Stirling Engine, 49th IEEE Conference on Decision and Control, Altanta, Georgia, USA (2011)

10. E. Garcia-Canseco, A. Alvarez-Aguirre, J. M. A. Scherpen, Modeling for Control of a Kinematic Wobble-Yoke Stirling engine, Renewable Energy, 75 (2015) 